\title{
Teaching Mobile Communication in an e-Learning Environment
}

\author{
Laura Bergström, Kaj J. Grahn, Jonny Karlsson, \\ and Göran Pulkkis \\ Arcada Polytechnic, Helsinki, Finland
}

laura.bergstrom@arcada.fi kaj.grahn@arcada.fi jonny.karlsson@arcada.fi goran.pulkkis@arcada.fi

\begin{abstract}
This article presents a virtual course with the topic mobile and wireless communication systems. The structure of the tree sections of the course material content, the generic technology section, the wireless technology section, and the mobility management section, is presented.

The main didactical approach of the virtual course is a guided excursion to which students enroll. The task sets, consisting of exercises and study directives, that the course teacher assigns each week to the students are described. The concept of step-by-step skill assimilation, which lies behind the student guidance process, is outlined together with descriptions of the different user skill levels. The course has been produced by Arcada Polytechnic as a part of a production team in the Finnish Virtual Polytechnic. The structure of the Finnish educational system together with the goals and structure of the Finnish Virtual Polytechnic are presented. The course development process is described in detail together with the software tools used to produce the course material. The graphical design of the learning platform is presented. The communicating dimension, the interface, and the esthetical dimension, and the layout are presented and analyzed in depth.

The IT infrastructure needed to implement and use the learning platform of the course is described and assessed. Issues like how the students are registered and authenticated to the course are presented together with the tools for communication and interaction between student and teacher. General IT requirements together with specific both server (course provider) and client (student) side IT requirements are presented. Teaching and learning experiences, gathered from a wireless networking course held in spring 2005, are presented. In this wireless networking course Mobile IP animation was used to simplify both teaching and learning of mobile IP. Further development needs are also presented.
\end{abstract}

Keywords: didactical approach, e-learning, graphical design, mobile, platform, wireless.

Material published as part of this publication, either on-line or in print, is copyrighted by the Informing Science Institute. Permission to make digital or paper copy of part or all of these works for personal or classroom use is granted without fee provided that the copies are not made or distributed for profit or commercial advantage AND that copies 1) bear this notice in full and 2) give the full citation on the first page. It is permissible to abstract these works so long as credit is given. To copy in all other cases or to republish or to post on a server or to redistribute to lists requires specific permission and payment of a fee. Contact Publisher@InformingScience.org to request redistribution permission.

\section{Introduction}

Wireless communication technologies provide significant advantages compared to wired technologies. A wireless networks eliminate the need for network cables since wireless radio interfaces are accessed over the air. Wireless networks also provide support for mobility, which means that a moving device can remain 
network connected also while the network access point changes and even when the access network type changes. The evolution of wireless technologies and mobility management schemes is currently advancing rapidly. Existing networking services can be offered on mobile communication platforms and the availability of mobile communication platforms also makes new network service types possible. (Pagani, 2005)

Wireless and mobile networking is thus an important and highly relevant topic for IT education in universities and polytechnics. Arcada Polytechnic offers IT engineering education on Mobile and Wireless Communication Systems also in an e-learning environment.

\section{Course Content}

The course content consists of three structured sections: Generic Wireless Technology, Wireless Technology Types, and Mobility Management. These sections can be found from a navigational menu on the course portal. In the menu there are also links to the course index, all the exercises and the weekly topics.

\section{Generic Wireless Technology Section}

The general protocol architecture for mobile networking is outlined. Radio interfaces in wireless networking are described. Quality of Service (QoS) of a network and QoS management are characterized. Modulation and access methods are described.

\section{Wireless Technology Type Section}

Wireless communication technologies are described according to the following taxonomy (See Appendix for list of abbreviations):

- Wireless Cellular Network Technologies

- GSM evolution based technologies

- $2 \mathrm{G}$ (GSM, HSCSD, GPRS, EDGE, EDGE Evolution)

- $3 \mathrm{G}$ (UMTS, HSDPA, HSUPA, HSPA+, SAE/LTE)

- $4 \mathrm{G}$

○ other wireless cellular network technologies

- MBWA

- Flash OFDM

- Wireless Network Technologies Classified By Coverage Range

- Wireless PAN Technologies

- IrDA

- UWB

- RFID

- Bluetooth

- Wibree

- Zigbee

- Wireless LAN Technologies

- WLAN

- Wireless MAN Technologies

- WiMAX

- Wireless ATM

- Wireless WAN Technologies

- Satellite Communication

- GPS 
Each wireless communication technology is described by

- An Introduction

- Underlying Standards

- System Architecture

- Radio Interface and Modulation

- Protocol Architecture

- Quality of Service (QoS) Issues

- Security

\section{Mobility Management Section}

Networking mobility types are terminal mobility, application mobility, and identity mobility. Terminal mobility or node mobility means that a terminal or network node moves to another location or to another network domain with preserved network connectivity. Application mobility means that a software process moves to another host node. Software agent technologies are typical implementations of application mobility. Identity mobility means that an identity defined as a name, a number, or cryptographic key moves to another location or to another computer. (Candolin, 2005)

In this section only the case of terminal mobility or node mobility will be considered. A mobility management scheme for node mobility must solve the following problems:

- the node location problem, to find the current point of network attachment

- data transfer to and from the current node location

- continuation of data transfer after the node or the network has moved

- controlled disconnection of a node from the network

- performance optimization, for example minimization of the network load of a mobility management scheme.

Node mobility categories are

- nano mobility, the network node moves to another location but the point of attachment doesn't change

- micro mobility, the network node moves to another location and the point of attachment changes within the same network domain

- macro mobility, the network node moves to another location and the point of attachment to another network domain. (Candolin, 2005)

The wireless technologies presented in the wireless technology section of the course support nano mobility and micro mobility. 2G/3G wireless cellular network technologies also support macro mobility, if all points of attachment are in interconnected $2 \mathrm{G} / 3 \mathrm{G}$ wireless cellular networks when a network node moves. Only the wireless cellular 4G technology, which is still a research vision, will support also macro mobility for different wireless access network technologies. This section of the course therefore contains macro mobility management scheme descriptions. These descriptions are structured according to their layer in the network protocol stack:

- Network Layer Mobility

○ Mobile IP

- MIPv4

- MIPv6

- IPSec Mobility MOBIKE

- LINA

- Transport Layer Mobility

- Stream Control Transport Protocol

- Extended Transport Control Protocol 


\section{- Migrate TCP}

- Application Layer Mobility

○ SIP

- Hybrid Mobility Schemes

○ HIP

○ Homeless Mobile IP

\section{Didactical Approach}

The chosen didactical approach is to offer the following two options to course students

- a guided self study process based on network access to the course portal and on guidance over network connections

- a guided excursion to which students from different polytechnics enroll.

In both cases a team consisting of a responsible teacher, a course assistant, and a graphical designer, the maintainer of the web based learning environment, provides the guidance.

\section{Guidance}

The starting point is that the course students are assumed to have basic networking knowledge and skills for wired networks. The course guidance is based on step-by-step knowledge and skill assimilation, starting from user level knowledge and skills.

\section{Course Material}

The course material consists of

- the course portal

- literature and web resources in course portal sections and subsections.

For a key issue of mobile networking, Mobile IP, a learning object has been designed as a structured animated network environment in which the communication between a $\mathrm{CN}$ and a $\mathrm{MN}$ can be studied. The communication logistics for all options of Mobile IP can be studied on the highest level. Communication details, like the data packet header fields, can be interactively studied on lower levels of the animated environment.

\section{Weekly Task Sets}

The course proceeds with weekly task sets assigned to the course participants using the web based course portal and the course mailing list. The weekly task sets consist of study directives and assignments. Assignments are configuration, installation, calculation, testing or programming exercises or topical quizzes. For example,

- a Mobile IP exercise consists of installation of Mobile IP client software, roaming tests, and an exercise report.

- a calculation exercise in wireless cellular networking is

$\circ$ If a signal-to-interference ratio of $16 \mathrm{~dB}$ is required for satisfactory forward channel performance of a cellular system, what is the cluster size that should be used for maximum capacity if the path loss exponent is (a) $n=3$, (b) $n=4$ or (c) $\mathrm{n}=5$. Assume that there are 6 co-channel cells in the first tier and all of them are at the same distance from the mobile. Use suitable approximations

- a wireless cellular networking quiz is a set of questions about the evolution of $3 \mathrm{G}$ wireless cellular networking (UMTS -> HSDPA -> HSUPA -> HSPA+ -> SAE/LTE) 
Each weekly task set has a deadline. The exercises assigned in the weekly task sets are included in the course portal.

\section{Elective Home Work Assignments}

Six elective home work assignments are given to the students. The tasks are to write and hand in short essays about current mobile communications issues. After accomplishing these tasks the student will get additional points added to the examination result.

\section{User Level Skills}

Every user of computers and computer networks needs certain mobile networking knowledge skills in using mobile services:

- how to obtain network access in a wireless access network

- authentication to a mobile network service with passwords, cryptographic tokens, and biometric methods

- use of X.509 certificates

- to check if a mobile service is authentic

- to create digital signatures required by mobile services.

\section{Administrator Level Skills}

The next level of mobile networking skills is the network administrator level, which should include

- skills to install, configure, secure, maintain, and update wireless access networks and mobile networking software/hardware

- network user support and training skills in mobile networking related issues.

Education of IT engineers and other IT professionals should provide network administrator level skills in mobile networking.

\section{Developer Level Skills}

The highest level of mobile networking skills in polytechnic education is the developer level, which is required in further development of mobile networking technology and in the implementation of new mobile services. On this level advanced programming, hardware design, wireless networking, mobility management, and network security skills are needed. Education of software and network engineering professionals should provide developer level skills in mobile networking.

\section{Scientific Level Skills}

The highest level of mobile networking skills in university education is the scientific level. This level covers knowledge and skills

- to propose new mobile networking protocols and standards.

- to propose new wireless access network types

- to further develop mobility management methodology.

It should be possible to acquire this skill level in postgraduate IT education in universities. 


\section{Course Development}

\section{The Finnish Virtual Polytechnic}

The Finnish educational system in a nutshell is illustrated in Figure 1. Compulsory basic education at comprehensive schools is given to all children between the ages of 7 and 16. Education is voluntary after completing the comprehensive school. Students may go to upper secondary school providing three years of general or vocational education. Both of these give a general qualification for polytechnic and university studies (The Finnish educational system in a nutshell, 2006); see Figure 1.

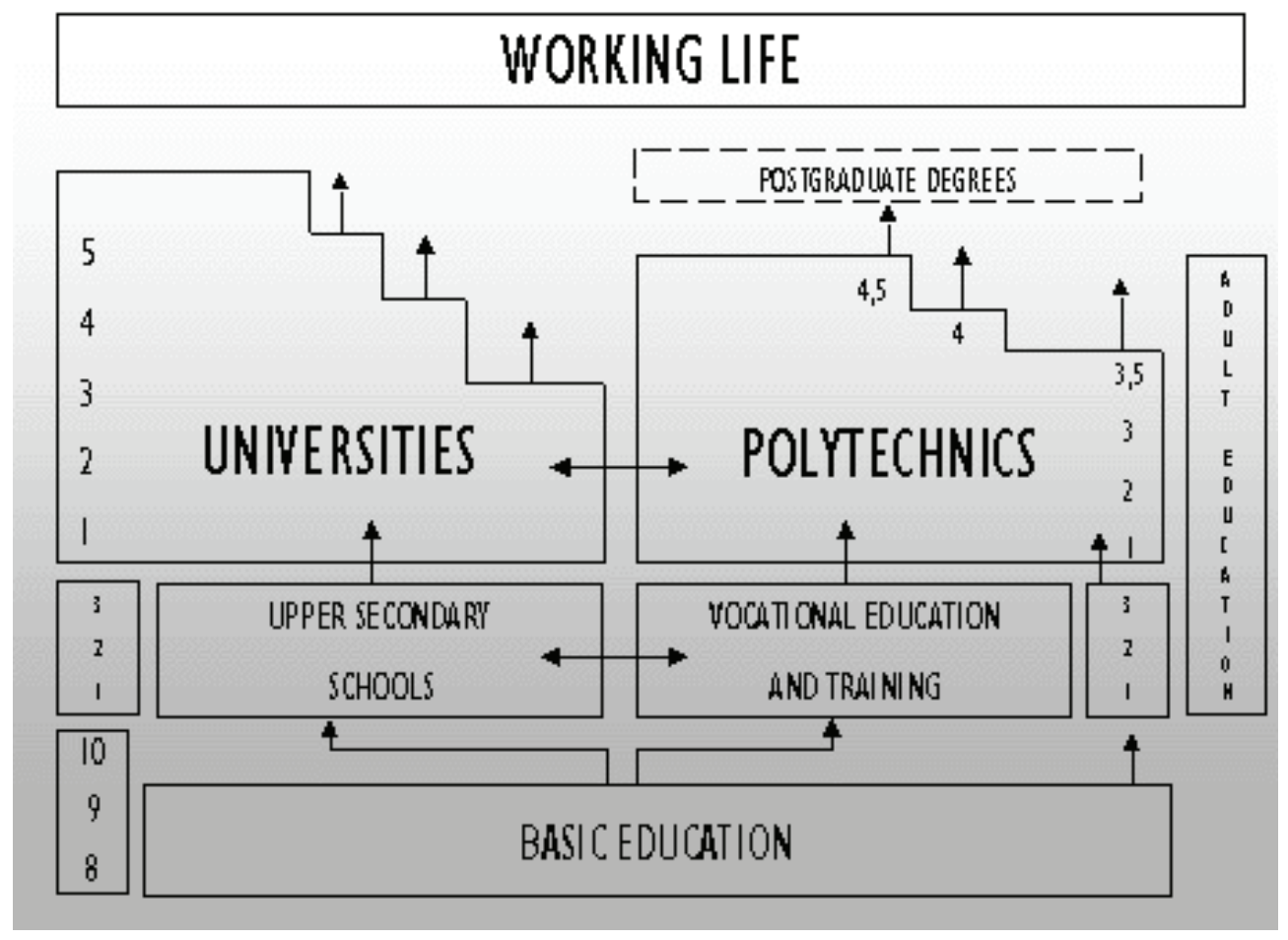

Figure 1: The Finnish educational system.

The Ministry of Education in Finland started in the years 2000 - 2004 the creation of a Virtual School, a Virtual Polytechnic and a Virtual University. Briefly, the goals for the Finnish Virtual Polytechnic are (The Virtual Polytechnic of Finland, 2006):

- Increase co-operation between polytechnics

- Increase the knowledge of virtual learning

- Build up a common portal for all students in Finnish polytechnics

$\circ$ for different areas of expertise

- provide education over networks

○ be aware of technical development (Digital TV, mobile Internet)

- Co-operation with other projects

○ locally and internationally

- Produce contents in co-operation with all polytechnics and other institutions

- Quality assurance

- Copyright questions (teacher - institution) 
- Standardization

○ Learning Platforms

- Materials, courses, material modules, meta-data

- Student administration

- Economical aspects

- Support for teachers who are producing material

Teacher education covers new skills like coaching students through learning environments on a net platform. Virtual learning is crossing borders not only between polytechnics but also to other schools and universities, and to other nations.

\section{Content Production Teams}

The Finnish Virtual Polytechnic has 31 polytechnics as members and a potential of 120000 students and 6000 teachers. During the period $2004-2006$ content production is being done in about 50 production teams including about 500 team members. The aim is to have virtual courses of more than 750 credit units (ECTS). The Mobile Communication Systems course has been produced in the production team Planning, Programming and Production of Mobile Services. The total amount of credit units in this production circle is 14 ECTS.

\section{Course Development Process}

Text and table based information has been produced by teachers and students. Figures, animations, and other graphical material production have been supported by other expertise within the polytechnic. The production team consists of 2 IT teachers, 1 IT research engineer and 1 graphical designer and 2 IT students. The efforts needed to develop the course included:

- The IT teachers and the research engineer have worked 4-5 hours/month during a 10 month period to plan the course and the content production, and to supervise the students and the graphical designer

- The IT students have worked about 20 hours/month during 6 months with content production for the course.

- The graphical designer has worked half time during about 6 months with

0 the web based learning environment

$\circ$ the Flash animations, and with

- picture design for the course content.

Course development continues during the study process of an accepted group of course students:

- Weekly tasks and given exercises are integrated in the web based learning environment

- The course schedule is updated every week

- Feedback and comments from course participants as well as response of the course teacher to this feedback is promptly published on the learning environment

- Course content is updated and revised based on the experiences from the ongoing course. For the update work the graphical designer is needed about 10 hours/week to support the course teacher.

Course material is produced using:

- Microsoft Word (.doc), FrontPage or Mozilla Composer (.html) for text

- Adobe PhotoShop and Macromedia Flash for pictures (.gif, .jpeg)

- Macromedia Flash for animations (.swf)

The course material has been organized in modules. Course testing and evaluation is done by the production team, by IT teachers, and by students who will use the course material. Accessibility and navigation have been tested using IE, Mozilla and Firefox browsers. 
This course, Mobile Communications, will be conducted for the first time in period 3, during spring 2008.

\section{The Graphical Design of the Learning Environment}

\section{Background}

Donald A. Norman (Norman, 2002) has identified two temptations for the designer that lead toward products that drive users to distraction: "Creeping featurism", which is the tendency to add the number of features, that a product or element can do, and "The worshipping of false images", which he explicit in false images like complexity. Regarding the first aspect, it's very important to keep the design of the elements in the learning platform simple, avoid features that are not absolutely necessary and organize these features with care. The second aspect solicits to ask your self which is $m y$ false image. In our case the false image could be the worshipping of a preestablished image on how a learning platform should be structured and designed. This image is partly created by affection to a previous design. Therefore, to avoid the creation of this image, should we ask ourselves if it is motivated to re-use a previously designed graphical interface and its organization model or should changes and improvements been made to it? Therefore we will analyze briefly the comprehensibility and usability of the learning platform "Network Security" (Bergström et al., 2004) in the purpose to achieve an improved design, an enhanced organization model, and a better usability of the learning platform "Mobile and Wireless Communication Systems". This operation focuses on the elements that can be improved; because only by individuating the weaknesses in a design can we turn them into its strengths.

First of all, to the two areas of the learning platform (course material and learning platform) are not given their needed attention in the graphical interface of "Network Security" (see Figure 2). Their difference should be highlighted and supported by the graphical design. These areas can be defined as the two states of the product and should therefore be evidenced to render clearer the alternatives for action of the user (visibility, one of Normans' principles of good design (Norman, 2002)). Also the placement of the elements in the environment should be better divided between these two areas (referring especially to "weekly topics" and "sources"). The areas have in fact different functionalities and specific roles, as the course material-area is a "container" of the didactical material and the learning platform-area is a "board" through which the teacher communicates with the students, and the placement of the elements should be planned in coherence with these roles.

The question of the importance of evidencing a specific aim of a certain element rises also when analyzing the content of the pages in the environment. For an easy navigation and comprehendible organization a page should have only one function or content (for example pdf-files for printing should all be gathered on one page, not spread around in the material). The only exception for this rule is the homepage, as the user will inevitably find this page and therefore the information in it. Because of this characteristic (frequent visits) on the homepage should be placed (only) information that is often read and updated. This aspect is not fulfilled in the "Network Security" environment as on the homepage you can find a lot of information that is not relevant for frequent users (see Figure 2).

It is important to plan the environment so that all the pages that are frequently visited are behind the fewest possible hyperlinks. In the aim of fulfilling this rule emerges the problem of the function "topic of the week" as each week the most read pages changes. Is it sufficient with hyperlinks to these pages from the homepage? This means however that you always have to return to the homepage (from, for example, a page in the course material) for an easy access to this material. Therefore, a better solution should be found for a quick access to the material "studied now". 
Other enhancements should be made regarding the organization of the course material, or more in particular evidencing its different parts with graphical enforcements (better menu of the course material). Also the text size should be 12 points (not 10) for an easy reading. Finally, the functionality of the course material index is under-used (the course material is listed as a table of contents with links, but without links to figures or tables), and should be updated to a more complete index drawing advantage of the fact that the course material is actually in HTML language (hyperlinks and bookmarks).

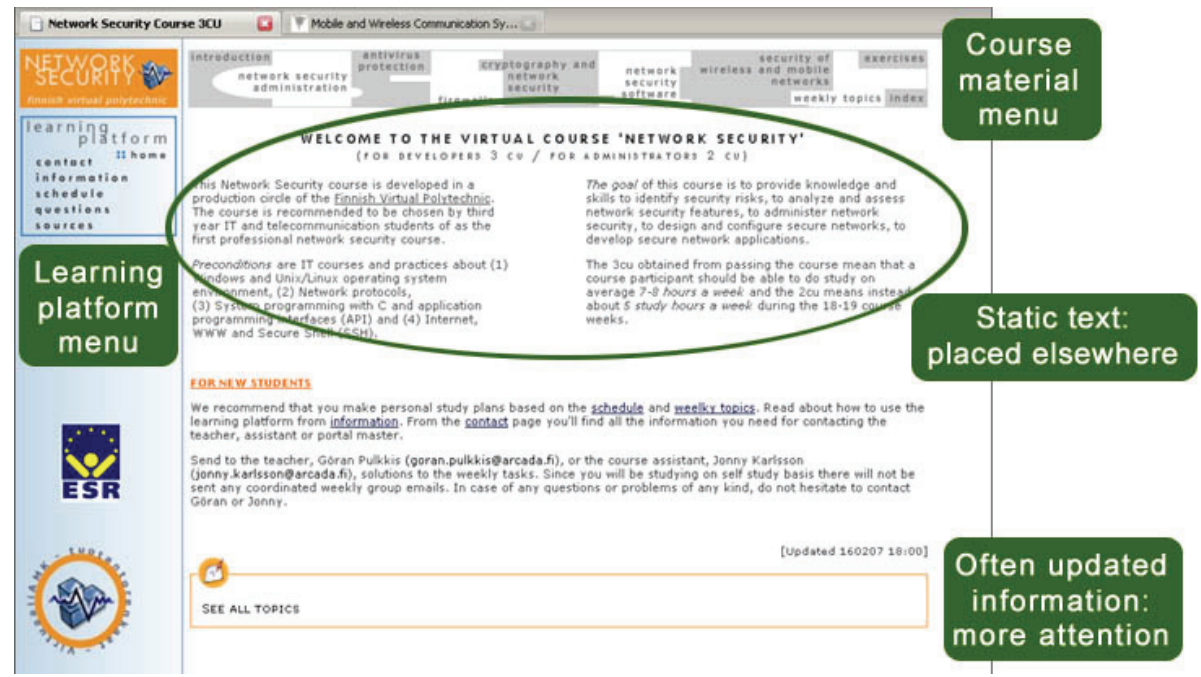

Figure 2: The homepage of our previous learning environment "Network Security". Its graphical interface is briefly analyzed in the purpose to explain the improvements that has been made in the design of the graphical interface of "Mobile and Wireless Communication Systems". The two menus, course material on the top and learning platform on the left, represent the ways of access to the two different areas of the environment.

These above mentioned aspects would need improvement in the "Network Security" environment, and represent therefore the guidelines for a future design. These are in fact the elements that we'll particularly take in consideration when planning and developing the graphical design of the learning environment "Mobile and Wireless Communication Systems".

Previous studies (Bergström et al., 2004) have shown that a graphical design is structured into two dimensions, a communicating dimension and an esthetical dimension. The communicating dimension, the interface, describes the interaction between the user and the learning environment. The esthetical dimension, the layout, describes the visual style of the whole website. As communication goals are more easily achieved with a strong esthetical structure (Mullet \& Sano, 1995), these two dimensions are very much dependent of each other and only together they make the learning environment good.

\section{The Interface}

The good interface aims to help the usability and comprehensibility of a product, and should support the user to achieve the goal that is set for using the product. In this case the goal is to learn, which makes the design of the interface even more important. The interface should let users be able to spend their full cognitive resources on the task, not on learning how to use the interface it self. This means that the design should be carefully planned throughout the entire environment. Therefore it's important to keep in mind, during the designing process, the four principles of good design (Norman, 2002). (1) Visibility means that by looking the user can tell the state of the de- 
vice and the alternatives for action. With (2) a good conceptual model there will be consistency in the presentation of operations and results and a coherent and consistent system image. Furthermore, with (3) good mappings it's possible to easily determine the relationships between actions and results, between controls and their effects. And finally a full and continuous (4) feedback about the results of actions will give support to the other three points.

\section{The Usability}

The three fundamental elements of usability are clarity of the navigational system, an articulated index and visibility of the elements of interaction between student and teacher. These elements are also included in the list of aspects that need improvement in the "Network Security" and get therefore special attention and thorough consideration in the design of the interface.

The navigation in the learning environment. Because of the individuated floe (not sufficiently evidenced the two areas of the learning environment) in the graphical interface of "Network Security", the learning environment "Mobile and Wireless Communication Systems" is divided into two color-coded areas. An orange learning platform area on top and a blue course material area underneath the first mentioned (see Figure 3).

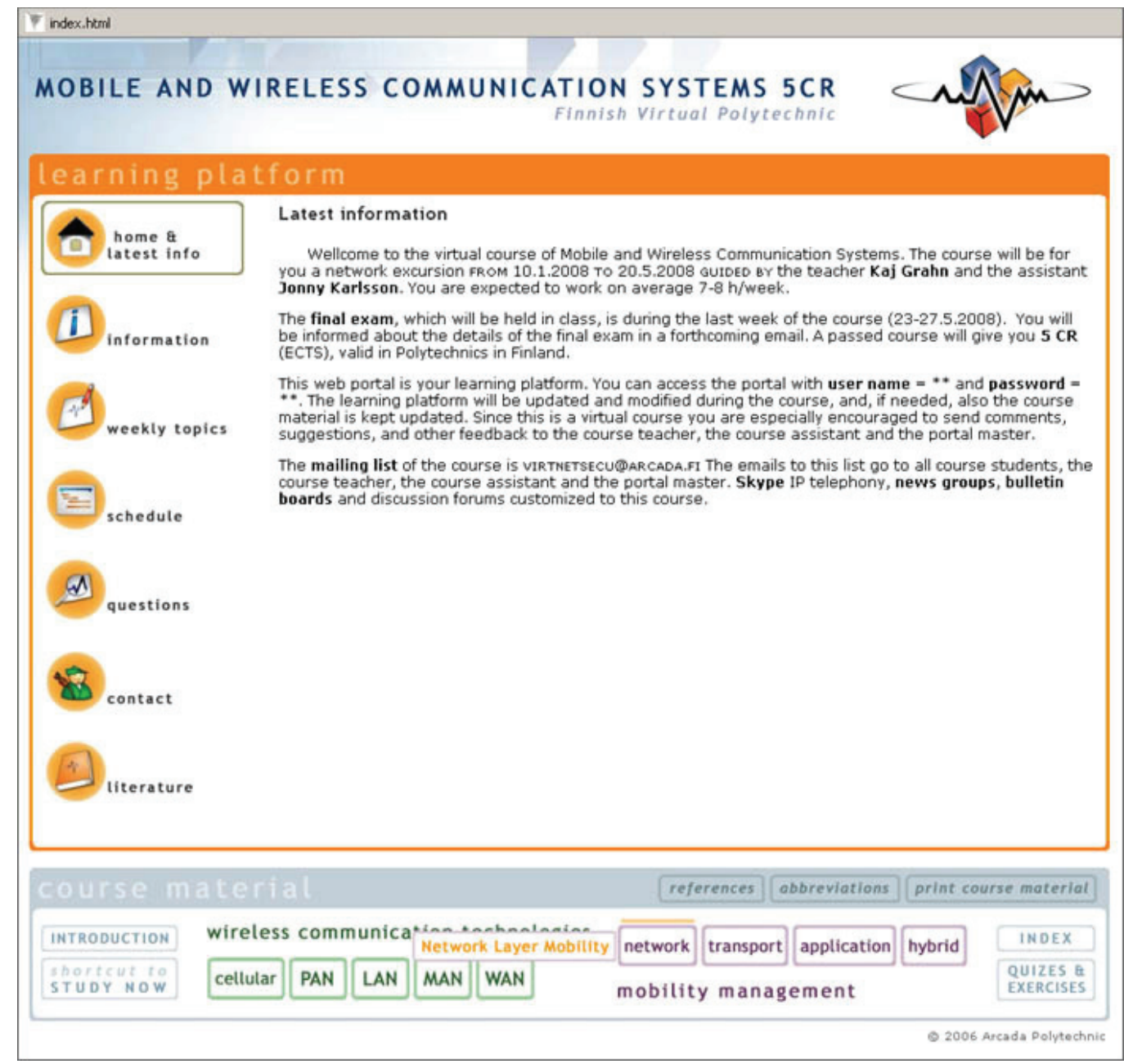

Figure 3: The homepage of the learning environment "Mobile and Wireless Communication Systems". The mouse is over the section "network" in the section mobility management of the course material. 
This rigid graphical division of the functionalities in the environment aims to clarify the two different states of it and therefore add visibility the alternatives for action. Both areas have independent menus. This two-menu system is in fact the fundamental aspect of the environments structure. In the orange area the user will find communications from the teacher/s and also all the information related to using the learning environment and the course it self. This area is therefore called learning platform. The blue area on the bottom is reserved for the course material and all topics related to it (references, abbreviations, print material, course material index and exercises).

A particular attention is given to the placement of elements in the interface. In the previous design all references where placed in the learning platform menu together with the list of the course literature. Now the references have been moved to the course material area, as these are in fact the bibliography of the didactical material. In the learning platform area the student can still find all the information regarding the course literature. Also the element "weekly topics", which in the last design was placed in the course material menu, is now moved to the area it belongs to, in other words to the learning platform menu. In the course material menu you can now find some new elements. In addition to the function "references", new elements of particular importance are "print course material" and "shortcut to study now". The first element brings the user to a page where all print material is gathered together, and the second one resolves the problem (that we brought up in the background paragraph of this section) of a quick access to the material studied that week. Using this link, which is always visible, the user will be transferred to the page in the study material that is to be studied that specific week.

When a learning platform area page is open, the course material area is reduced to its minimum (only the menu is visual) (see Figure 3), meanwhile when a course material page is open the learning platform menu changes from vertical to horizontal and only the horizontal of the orange learning platform menu is visual (see Figure 4). In this way the two menus are visible at all times. Furthermore, a selected page opens in its own area, in other words, if a page is selected from the course material menu it opens in the blue area and if it is selected from the learning platform menu it opens in the orange area.

To enhance the organization of the course material, and evidencing the different parts in it, the course material menu is designed to reflect the two main sections of the course. In other words, these sections, "wireless communication technologies" and "mobility management", are given their needed attention using a specific layout. Both sections have own color-coding, which reflects also their sub and sub-submenus that are the same color as the section they belong to (see Figure 4). The course material menu is structured of five "wireless communication technologies" topics and four "mobility management" topics, in addition to introduction, exercises and other elements that are connected to the course material but are not parts of it (for example index). All the contents of these nine main topics are organized in categories, or in a submenu that is always situated on the left underneath the main menu, inside a rectangle. If a category still has a submenu of its own, this will be placed before the long passages of text on the right. The navigation to subsection 'System Architecture' of the cellular wireless technology GSM is shown in Figure 4.

Index. The user should find the index button easily and fast, without having to select many hyperlinks, as this page will probably be an often-visited page of the user. Therefore it's positioned in the course material menu right next to the main topics and given, using the graphical design, the same degree of importance as are given for "introduction" or "exercises" buttons (form, color and size). The improved functionality of this index (compared to the one in the "Network Security" environment) consists of links to figures and tables in the course material. Moreover shortcuts to all the topics of the course material can be found here. 


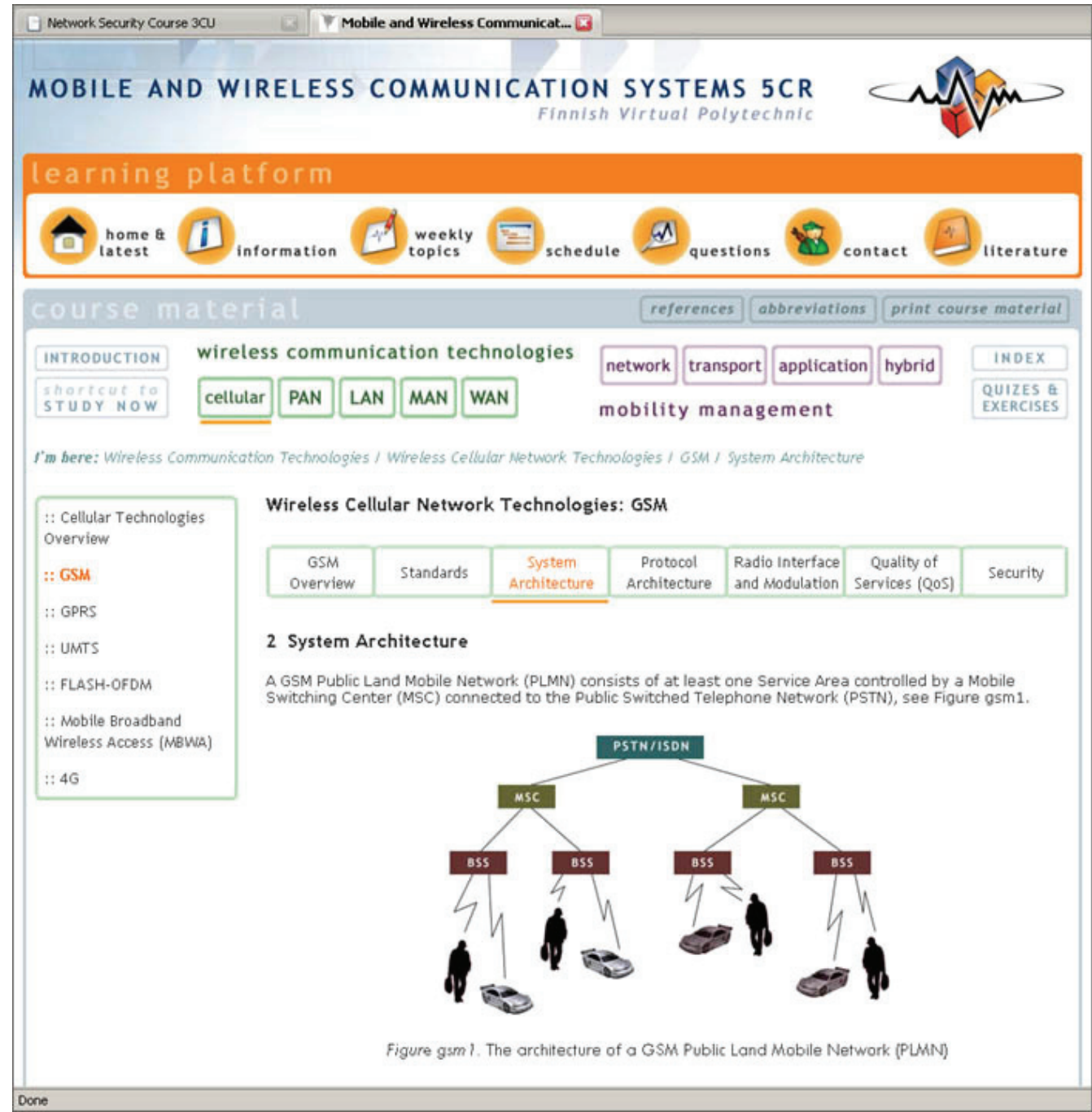

Figure 4: Navigation to subsection 'System Architecture' of the cellular wireless technology GSM.

Learning platform. Because of the importance of the elements in the learning platform area (they represent information widely used throughout the course) the clarity of its contents is enhanced by images (retrieved form the Finnish Virtual Polytechnic) and to its menu is given a better visibility (it occupies much more space in this interface than in the previous one). The information on the homepage changes week after week, and is therefore the most active element of the learning environment. Given its importance, to avoid an unorganized and distracting design on this page, the amount information on it is reduced to its minimum. As it contains only important notices or urgent information, in addition to the topics of the week, this often-updated information will now get all the attention it needs. All the other information that is addressed to the students (also the "static" information that in the previous environment was placed on this page, see Figure 2), he/she will find under the following links of the learning platform menu:

- A link to information about the conferencing area, bulletin board and newsgroups of the course, and a list of IT requirements that need to be fulfilled to be able to follow the course. On this page is also explained the two menu system.

- A link to the weekly topics page.

- A link to the course schedule outlining the significant events of the course. 
- A link to a questions page where all the questions and feedback that the students give during the course are gathered.

- A link to a page with the contact information of the teachers, assistants and other students.

- A link to the course literature and other resources.

\section{The Comprehensibility}

Returning to the four principals of good design, we'll now take them in consideration one by one. To give the elements their needed visibility that leads to clear alternatives for action, we need to organize them in a rational way. According to the laws of Gestalt the visual area is segmented into parts that assume a particular role (Appiano, 1993). These parts become objects or figures, meanwhile the background remains less concrete, thanks to the fact that the area is seen as a whole and the different parts in it are connected to each other by particular laws of organization. Some of the principal factors, which contribute to form a configuration/figure in a visual area, are proximity, resemblance and closure. These are in fact the three modalities for organization used in the interface. The learning environment is divided into two parts by closed rectangular areas, which frames are identical; closure separates the two areas from each other meanwhile resemblance re-establishes a connection between them, enhancing their equal importance. As a matter a fact, this kind of organization aims to point out the equivalent importance of these two areas to the functionality of the environment, not only through a layout, but also through size as, when the areas are reduced to their minimum, they are equally big. This is one more aspect that has been improved in this design compared to the previous one. Regarding the menus, the learning platform menu is organized by resemblance (six equally structured elements), and the course material menu by the use of all the three principals mentioned above.

For a good conceptual model it is important to have a consistency in the presentation of operations and results. This emerges above all through the structure of the pages, which always is the same in the pages belonging to a one and a same area. However the structure of the learning platform pages differs from the ones in the course material area. Also the feedback of the users' actions is coherent throughout the whole environment. As mentioned above, the elements need to give feedback for any action made by the user. This means that, when a user rolls over a link with his mouse, the link should react in some way to the users action so that he recognizes this as a link and, most of all, the link should give the user further explanations regarding its functionality (if this is not obvious from before). This is why the feedback becomes especially important in the course material menu where the main topic buttons need further specifications (for example "Network" stands for "Network Layer Mobility", see Figure 3). All other feedback is given by a rectangular/line that appears around the link (this is in the case of a button) or by highlighting the word that is a link (when the link is in the form of text). For clarity all links to other pages in the Internet are opened in a new window. The Mobile IP animation, which is independent of the rest of the course material, is opened in a new window. The animation is then seen as a story of its own and can easily be managed on its own.

The last of Norman's principals for good design is good mappings. This is achieved when the signs used in the interface have an efficient relationship between the signifier (sign) and it's signified (referent). According to the threefold classification of Peirce the modes of relationship between sign vehicles and their referents are:

1. Symbolic/symbol: a mode in which the signifier does not resemble the signified but which is fundamentally arbitrary or purely conventional, so that the relationship must be learnt (for example the sign representing a hand when a mouse is brought on a link)

2. Iconic/icon: a mode in which the signifier is perceived as resembling or imitating the signified, being similar in possessing some of its qualities (for example the sign representing lady on a toilet door) 
3. Indexical/index: a mode in which the signifier is not arbitrary but is directly connected in some way (physically or causally) to the signified, this link can be inferred for example as natural sign (smoke is a sign of fire) (Veivo et al., 1999 and Chandler, 2005).

The learning platform menu has been richened with signs or images that are symbols (information), icons (schedule, contact and literature) and indexes (home and weekly topics), in addition to signs in the form of language (symbols). Instead in the course material menu all the signs are symbols (language in general). The reason to this is the difficulty in describing the elements in an efficient way with images. For example, if "print course material" was described with an image of a printer instead of these word, would the user understand that this opens a page where all the course material for printing is gathered or would he comprehend this button as a command for printing the visible page? Although correctly represented images are much more efficient than words, images that "almost" stand for their signifier (sign) can lead to confusion and are therefore avoided.

\section{The Layout}

The goal of the communication between the user and the course website is more easily achieved with a strong esthetical structure. Inviting colors, a sober font on a calm background, a clear and organized positioning of the elements can motivate the user to reach his goal (learn) and awake is interest towards the learning environment and its contents. Therefore the quality of the user interface is very important (especially when the goal of the user is to learn): the screen should always look like an organized workspace. Of course the opinion of what is aesthetically beautiful is personal, but a good rule is: simplicity is elegance.

Visual structure. Here we'll shortly introduce the three main principals of a good visual structure: unity, contrast and grouping (Mullet \& Sano, 1995). The unity of the pages (regarding structure, color, text size and more) and a coherent design of the elements create an elegant website. The same structure of the material, figures and tables is kept throughout the course portal. This means that the learning environment has a coherent structure of the submenus, the sub-submenus, the headers, and the text (see Figure 4). Contrast enhances the difference between separate elements in one area and it creates clarity and harmony in the entire layout. Therefore the two main areas of the course portal are color-coded into different colors (contrast) while still being united to each other because of the form and structure (unity). Contrast is also held in mind when planning the text area; it differs from other areas because it is the only "area" that is not inside colored rectangles (see Figure 4) and because there are no other long text-passages visible on the page. In fact, similarity in form can be interpreted as similarity in content and therefore mislead the user to think that two, although separate, text passages would be connected to each other in some way. This introduces a third important element of a good visual structure, grouping. Elements belonging together are grouped to balance the screen and also for them to obtain the property of one element instead of many elements together (the laws of Gestalt).

Colors. The biggest improvement that has been made in this environment compared to the previous design is in the brave use of colors. The goal of the color composition in this interface is in fact to organize the information and ease its comprehension. Therefore color combinations that wake attention has been chosen as colors help grouping and highlighting hierarchy. Nevertheless, to make the reading and concentrating easier, all background colors are white and large areas of strong colors that irritate the eye have been avoided: this is the reason why the course material area is light blue, and not orange like the learning platform area. These factors are especially important when designing a website to be used frequently by the same user.

The font type. An ultimate improvement that has been made in this new design regards the font type. The font type used in text passages has been changed from Verdana, dark gray and 11 
points to Verdana, black and 12 points. This renders the text more visible (color black) and easier to read (size 12). Verdana is a font without serifs (without end curves in the letters) and because of that it has a better legibility on the computer screen. Furthermore, when a word or a phrase is highlighted in a text, it is bolded instead of underlined, which should be explicitly used for hyperlinks or titles because of its strong convention. Long passages of unbroken text are avoided.

\section{The IT Infrastructure of the Learning Platform}

\section{Registration to the Finnish Virtual Polytechnic}

The registration process will be handled by the Finnish Virtual Polytechnic's student office, which will probably be an electronic online office. Once the users have registered, and received their study place, the Finnish Virtual Polytechnic will create an account for them. There are two choices of accessing this account; using standard username and password authentication or by using a PKI certified cryptographic key pair. The private key of this key pair and the cryptographic operations using this private key may be hosted on a smart card based electronic ID card. If the student has an electronic ID card, the student's SATU number will be registered and stored in a LDAP directory. The SATU number is a unique public personal code in a Finnish electronic ID card (FINEID, 2006).

\section{Authentication}

Once the course participants have successfully applied and registered to the Finnish Virtual Polytechnic they will be authenticated and granted access to the learning environment of the network security course, hosted by a web server, a news server, and SSH servers. Authentication is preferable achieved using a Finnish electronic ID card, a FINEID card (FINEID, 2006). Anyone permanently living in Finland can apply for a FINEID card. Any granted web server can look up the access information stored in the LDAP directory, hosted by the Finnish Virtual Polytechnic.

\section{Communication}

In the real world, like in a class in any normal university or polytechnic, communication is a very important part of the learning process. The students have to be able to interact with the teacher, and with each other. Students need to exchange information by establishing a fruitful dialogue. Therefore, it is important that an online course can provide these same conditions. Students need to be able to exchange information with each other, even though they may be geographically scattered over a big area. The communication described above will be established using four different techniques: email, newsgroups, real time chat, and VoIP (Voice over IP) functionalities.

\section{Email}

The course will have a mailing list hosted by a majordomo server. When students register to the course they will also be registered on a mailing list. This mailing list is used for sending out information to all course participants, for instance topical study directives, exercises, examination dates, etc. It may also be used to distribute urgent information, since every student attending the course will receive a copy of the emails sent to the list. Emails can of course also be used for direct communication between student and teacher or between students. 


\section{Newsgroups}

The course will have a newsgroup where the participating students can discuss topics related to the course. This is the main forum for the students. The information sent to the newsgroup should not be urgent, for urgent information it is preferable to use the mailing list. An un-moderated newsgroup is very suitable for creating the communication environment mentioned earlier. Since the news system is threaded, it is very easy to navigate between the articles found in the newsgroup. Each new subject will be a new top-post and all comments concerning this subject will be added as a follow-up to that specific thread. The message board of the course is another moderated newsgroup. It is up to the student to check the newsgroup for new information independently. The students will not be notified when there is new information available in the newsgroups.

\section{Real Time Chat and VolP}

It can sometimes be hard to have a serious dialogue with someone using email or newsgroups. If you need answers to your questions fast, and if you have resulting questions, it is preferable to use real time communication. Especially when there are many people involved in a discussion, it is much easier to have a real time chat or a voice conversation. Real time chat functionality and voice conversations will be accomplished by using Skype (Skype, 2006).

Skype is a proprietary peer-to-peer VoIP network. Skype users can make voice and video calls through a computer equipped with Skype software and Internet connection. Skype also supports real time chat. Both voice and chat communication between Skype software users is free. Skype users can also communicate with mobile and regular landline phones but such calls are however not free. The Skype software is currently available free of charge and can be downloaded from the official Skype portal (Skype 2006).

\section{IT Requirements}

The course is intended to be an online course. The start version of the web based course portal can also be distributed on a CD instead of being hosted by a web server. For online viewing, a permanent high bandwidth (or broadband) Internet connection is recommended. However, it is possible to view the course using an ISDN line or even a modem dial-up connection. The part of the course containing a flash animation will of course not load very fast on a dial-up connection. However, most parts of the course are built on simple HTML pages.

\section{Server side}

The course is hosted by a web server, preferable by an Apache (Apache, 2006) server running on Linux, with integrated support for SSL (OpenSSL, 2006) and smart card authentication. The Finish electronic identity card (FINEID) can be used to identify users and to authenticate them. The access information has to be looked up in an LDAP directory (OpenLDAP, 2006). If the authentication is successful, the user will be granted access to the web server. The web server needs proper configuration for the PKI (or smart card based) authentication to work. However, old fashion password authentication is also possible to use.

\section{Client side}

The user needs a new version of a web browser with a Flash Player in order to correctly view the course. Internet Explorer provides the best support for the course layout. To be able to hear the audio in the animations, a standard sound card and loudspeakers are needed. 
For communication purpose, the user needs an email client and additionally also the Skype software, loudspeaker, and microphone for real time communication. To read the conferencing area and message board of the course a news client is needed. The news client is often integrated in the email client. Access to the online course will only be given to students registered at the Finnish Virtual Polytechnic.

\section{Experiences and Evaluation}

The Mobile IP animation was included in the course Wireless Networks (5 ECTS). Totally 14 students enrolled in the course held in spring 2005. The course was a module of the specialization studies Wireless Applications (33 ECTS). These specialization studies are intended for persons with a BSc (Eng.) compatible or polytechnic degree in Computer and Telecommunication Engineering. The goal was to deepen and develop the participant's knowledge in Computer and Telecommunication Engineering.

\section{Evaluation of Mobile IP Animation}

The Mobile IP animation was evaluated as a learning object from a pedagogic and heuristic point of view by a team from the Finnish Virtual Polytechnic (Finnish Virtual Polytechnic, 2006). The evaluation team was randomly chosen among the members of the content production teams. According to the evaluation, the strength is in the context and in the authentic problem formulation. Also the fact that the user can focus on some details of the Mobile IP is considered particularly good. On the other hand, improvements regarding support for thinking and problem resolving processes of the user could be made. The team pointed out, that tasks could be associated to the animation in such a way that the user finds the answers simply by navigating in the animation.

The positive feedback regarding the heuristic evaluation of the platform is its simple navigation. An efficient and flexible interface offers the user an easy and controlled use of the platform. More user instructions before starting the animation were also wanted. According to the team, the visualization is expressive and clear. The introduction of the animation received most critics, because it is not clear and interactive enough. As positive factors, the team also mentioned the fast uploading of the platform and the fact that the platform works in may different environments.

\section{Further Course Development Needs}

Issues of further course development are:

- An automatic exercise approval system is included

- Ready-made special instructions are made to help the students solve the most common problems in every exercise.

- The implementation of audiovisual real-time communication to the course is under planning, because the communication between students and teacher using email and SMS-messages is insufficient. The technologies considered are point-to-point video communication, web casting, telephony and online chat.

- Further development is mainly based on feedback from the course students.

- Additional animation parts are under discussion.

The development of a course held on a virtual learning platform is a challenging task. The process has also given the involved students insight into the processes of designing and teaching a virtual course. It would have been impossible to design the course within the course timeframe without the contribution from the students involved. 


\section{Conclusions}

Wireless and mobile communication is currently a hot topic and it is thus important to include it in the education of computer and telecommunication engineers. The benefit of teaching wireless mobile communication in a virtual course is that students from polytechnics all over the country, and also from foreign polytechnics, can share and participate in the same course without being physically present. Since students from several different polytechnics participate, feedback and development suggestions are retrieved in a much larger perspective, compared to in an internal course, which results in a higher quality of the course material.

The production of a virtual course is, however, a much more demanding task than the production of a traditional internal course. Experts, like graphical designers, have to be included in the production team. The graphical layout of the learning environment is important in order to make the learning and browsing of the course material interesting and easy as well as to lead the students' attention to the essential parts. Before the course is in its final form many prototypes have to be tested and feedback from the students is needed. A proper choice of computer software and IT technology is necessary. A sufficient and realistic budget is also essential.

Teaching and learning in a virtual course is also more demanding than in an ordinary course. Students cannot directly interact with the course teacher in the same way as during lectures and teacher assisted exercise sessions. Email and newsgroups is not enough for assisting students since they don't provide direct communication. It is thus important to provide the students the possibility to use real-time E-communication channels such as text, voice, and video chat.

\section{References}

Appiano, A. (1993). Comunicazione visiva: Apparenza, realtà, rappresentazione. Turin: UTET Libreria Srl.

Bergström, L., Grahn, K., Karlström, K., Pulkkis, G., \& Åström, P. (2004). Teaching network security in a virtual learning environment. Journal of Information Technology Education, 3, 189-217. Retrieved March 11, 2007 from http://jite.org/documents/Vol3/v3p189-217-038.pdf

Bergström, L., Grahn, K., \& Pulkkis, G. (2006). A virtual learning environment for mobile IP. Journal of Issues in Informing Science \& Information Technology, 3, 83-101. Retrieved March 9, 2007 from http://informingscience.org/proceedings/InSITE2006/IISITBerg205.pdf

Candolin, C. (2005). Securing military decision making in a network-centric environment (Doctoral Dissertation, Helsinki University of Technology, Finland, 2005). Retrieved March 8, 2007 from Helsinki University of Technology, TKK Electronic Academic Dissertations Web site: http://lib.tkk.fi/Diss/2005/isbn9512279819/isbn9512279819.pdf

Chandler, D. (2005). Semiotics for beginners. Retrieved March 10, 2007, from University of Wales Web site: http://www.aber.ac.uk/media/Documents/S4B/sem02.html

FINEID. (2006). Population register centre. FINEID.FI site - technical information about the electronic identity. Retrieved December 6, 2006 from http://www.fineid.fi

Finnish Virtual Polytechnic (2006). Production team evaluation page (internal). Retrieved December 15, 2006, from https://www.virtuaaliamk.fi/opintojaksot/041005/1092991850017/1099044944687/1113319626141/11 $\underline{13319658781 . h t m l . s t x}$

Mullet, K. \& Sano, D. (1995). Designing visual interfaces. Mountain View, CA: Sun Microsystems, Inc.

Norman, D. A. (2002). The design of every day things. New York: Basic Books.

OpenLDAP Foundation (2006). OpenLDAP - community developed LDAP software. Retrieved December 6, 2006 from http://www.openldap.org 
OpenSSL. (2006). The OpenSSL Project. Retrieved December 6, 2006 from http://www.openssl.org

Pagani, M. (Ed.) (2005). Mobile and wireless systems beyond 3G: Manging new business opportunities. Hershey PA and London: IRM Press

Skype Limited (2006). The official Skype portal. Retrieved December 6, 2006 from http://www.skype.com

The Finnish educational system in a nutshell. (2006). Retrieved December 15, 2006 from http://www.tokem.fi/english/KVO/International_Exchange/The_Finnish_Education_System/etusivu.ht $\mathrm{ml}$

The Virtual Polytechnic of Finland. (2006). Retrieved December 15, 2006 from http://www.tpu.fi/virtuaaliamk/index eng tiedostot/v3 document.htm

Veivo, H \& Huttunen, T. (1999). Merkeistä mieleen ja kulttuuriin. Helsinki: Oy Edita Ab.

\section{Appendix: List of Abbreviations}

\begin{tabular}{|c|c|c|c|}
\hline $2 \mathrm{G}$ & Second Generation & LDAP & Lightweight Directory Access \\
\hline $3 G$ & Third Generation & \multicolumn{2}{|l|}{ Protocol } \\
\hline $4 \mathrm{G}$ & Fourth Generation & \multicolumn{2}{|c|}{ work Architecture } \\
\hline ATM & Asynchronous Transfer Mode & LTE & Long Term Evolution \\
\hline $\mathrm{CN}$ & Communicating Node & MAN & Metropolitan Area Network \\
\hline ECTS & European Credit Units & MBWA & Mobile Broadband Wireless \\
\hline EDGE & Enhanced Data rates for GSM & \multicolumn{2}{|l|}{ Access } \\
\hline Evolution & & $\mathrm{MHz}$ & Megahertz \\
\hline FINEID & Finnish Electronic Identity & MIP & Mobile IP \\
\hline Flash OFDM & Fast Low-latency Access with & MN & Mobile Node \\
\hline \multicolumn{2}{|c|}{ Seamless Handoff OFDM } & MOBIKE & Mobile IKE \\
\hline GPRS & General Packet Radio Service & OFDM & Orthogonal Frequency Divi- \\
\hline GPS & Global Positioning System & \multicolumn{2}{|c|}{ sion Modulation } \\
\hline GSM & Global System for Mobile & PAN & Personal Area Network \\
\hline \multicolumn{2}{|c|}{ Communications } & PKI & Public Key Infrastructure \\
\hline HSCSD & High Speed Circuit Switched & QoS & Quality of Service \\
\hline Data & & RFID & RF Identification \\
\hline HSDPA & High Speed Downlink Packet & SAE & System Architecture Evolu- \\
\hline Access & & tion & \\
\hline HSPA & High Speed Packet Access & SATU & “Sähköinen Asiointitunnus” \\
\hline $\mathrm{HSPA}+$ & HSPA Evolution & \multicolumn{2}{|c|}{ (means "Electronic Communication Code") } \\
\hline HSUPA & High Speed Uplink Packet & SIP & Session Initiation Protocol \\
\hline Access & & $\mathrm{SSH}$ & Secure Shell \\
\hline HIP & Host Identity Protocol & SSL & Secure Sockets Layer \\
\hline HTML & Hypertext Markup Language & TCP & Transmission Control Proto- \\
\hline ID & Identification & \multicolumn{2}{|l|}{ col } \\
\hline IE & Internet Explorer & UWB & Ultra Wide Band \\
\hline IKE & Internet Key Exchange & VoIP & Voice Over IP \\
\hline IP & Internet Protocol & WAN & Wide Area Network \\
\hline IPSec & IP Security & WiMAX & Worldwide Interoperability \\
\hline IrDA & Infrared Data Associations & \multicolumn{2}{|c|}{ for Microwave Access } \\
\hline IT & Information Technology & WLAN & Wireless Local Area Network \\
\hline LAN & Local Area Network & & \\
\hline
\end{tabular}




\section{Biographies}

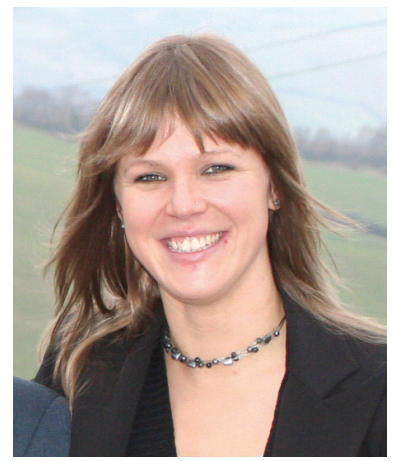

Laura Bergström has a BSc in Media Culture from Arcada Polytechnic, Helsinki, Finland. Since March 2002 she works for Arcada Polytechnic as graphical designer in virtual education development.

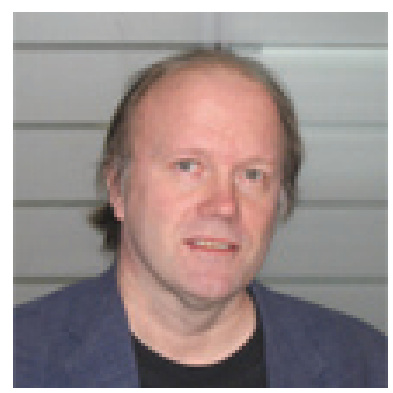

Kaj J. Grahn, Dr. Tech., is presently senior lecturer in Telecommunications at the Department of Business Administration, Media, and Technology of Arcada Polytechnic, Helsinki, Finland. He is also Program Manager of the Electrical Engineering Programme. His current research interests include Wireless and Mobile Network Security.

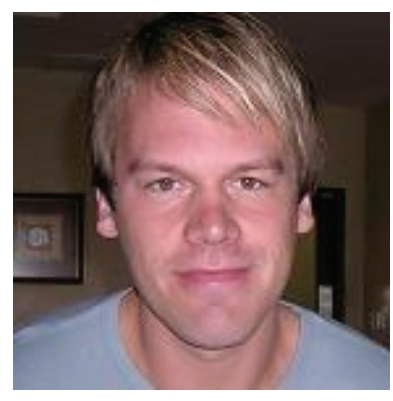

Jonny Karlsson has a BSc in Information Technology from Arcada Polytechnic, Helsinki Finland. Since May 2002 he has been working in Arcada Polytechnic as a course assistant and course teacher in programming and network security related courses and as a research assistant. His current research interests include Wireless and Mobile Network Security.

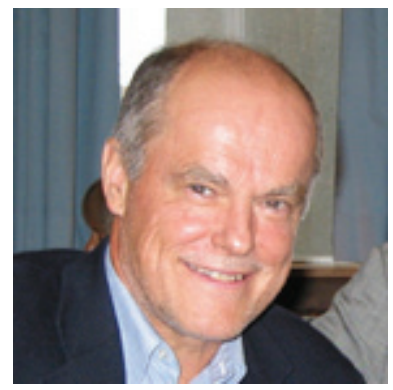

Göran Pulkkis, Dr. Tech., is presently senior lecturer in Computer Science and Engineering at the Department of Business Administration, Media, and Technology of Arcada Polytechnic, Helsinki, Finland. His current research interests include Network Security and Applied Cryptography. 